\title{
Publisher Correction: Approaches and advances in the genetic causes of autoimmune disease and their implications
}

\author{
Jamie R. J. Inshaw (D), Antony J. Cutler D, Oliver S. Burren, M. Irina Stefana and John A. Todd
}

Correction to: Nature Immunology https://doi.org/10.1038/s41590-018-0129-8 (2018), published online 20 June 2018.

In the version of this article initially published, the bibliographic information for reference 2 was incorrect in the reference list, and reference 2 was cited incorrectly at the end of the second sentence in the second paragraph (“...were identified ${ }^{2}$."). The correct reference 2 is as follows: "Kong, A. et al. The nature of nurture: Effects of parental genotypes. Science 359, 424-428 (2018)." The reference that should be cited at the end of the aforementioned sentence, which should be numbered ' 5 ' ("...were identified 5 ."), is as follows: "Okada, Y. et al. Genetics of rheumatoid arthritis contributes to biology and drug discovery. Nature 506, 376-381 (2014).” All subsequent references (5-161) should be renumbered accordingly (6-162) in the list and text. Also, several of the gene symbols in Table 2 were formatted incorrectly (without commas); the correct gene symbols are as follows: column 3 row 13, RBM17, IL2RA; column 3 row 30, DEXI, CLEC16A; column 3 row 39, UBASH3A, ICOSLG; column 4 row 15, PTEN, KLLN; column 4 row 21, CLEC7A, CLEC9A; and column 5 rows 7-9, AL391559.1, ENSG00000238747, RP11-63K6.7, RP3-512E2.2. The errors have been corrected in the HTML and PDF version of the article. 